\title{
An adolescent girl diagnosed with IgA nephropathy following the first dose of the COVID-19 vaccine
}

\author{
Mari Okada $^{1}$ (D) $\cdot$ Eriko Kikuchi $^{2} \cdot$ Masayuki Nagasawa $^{1} \cdot$ Akihiro Oshiba $^{1} \cdot$ Masuhiro Shimoda $^{2}$
}

Received: 30 September 2021 / Accepted: 23 December 2021 / Published online: 3 February 2022

(c) Japanese Society of Nephrology 2022

\begin{abstract}
There has been growing interest in reported cases of $\operatorname{IgA}$ nephropathy (IgAN) flare-up following administration of the coronavirus disease 2019 (COVID-19) vaccine. Our patient is a previously healthy 17-year-old girl who presented with a 10-year history of microscopic hematuria. Because there were no abnormal findings in blood examination or ultrasonography, we followed her up twice per year as asymptomatic hematuria. Although she never developed gross hematuria when she had upper respiratory infections or received an influenza vaccine, she presented with gross hematuria and proteinuria within a few days after receiving the first dose of the Pfizer vaccine. We performed renal biopsy 2 weeks after the first vaccination. It revealed minor glomerular abnormalities with diffuse mesangial IgA deposits, and we diagnosed her with mild IgAN. Gross hematuria was detected after both the first and second doses, although it changed to microscopic hematuria within 1 week. Additionally, her proteinuria resolved spontaneously approximately 10 days after the second dose of the vaccine. Therefore, we opted to observe her without administering medication. The causation between COVID-19 vaccination and IgAN flare-up remains unclear. Several reports showed IgAN patients presenting gross hematuria following the second dose of the Pfizer or Moderna vaccines. However, our patient developed gross hematuria and proteinuria even after the first dose and without known severe acute respiratory syndrome coronavirus 2 exposure. Nephrologists should inform both patients with IgAN and those with asymptomatic hematuria that this side effect can occur even after the first vaccination.
\end{abstract}

Keywords IgA nephropathy $\cdot$ Adolescent $\cdot$ COVID-19 $\cdot$ SARS-Cov- $2 \cdot$ Vaccine

\section{Introduction}

Several cases of IgA nephropathy (IgAN) flare-up following administration of the coronavirus disease 2019 (COVID19) vaccine have been reported as the vaccinations progress worldwide. The Ministry of Health, Labour, and Welfare in Japan approved the BNT162b (Pfizer-BioNTech) and mRNA-1273 (Moderna) COVID-19 vaccines for individuals aged over 12 years old on June 1 and August 3, 2021, respectively. A previous report described two pediatric cases of IgAN presenting as gross hematuria after receiving the second dose of the Pfizer COVID-19 vaccine [1]. Herein,

Mari Okada

okada-mr@musashino.jrc.or.jp

1 Department of Pediatrics, Musashino Red Cross Hospital, 1-26-1, Kyonan-cho, Musashino-shi, Tokyo 180-8610, Japan

2 Department of Pediatrics, Nerima Hikarigaoka Hospital, 2-11-1, Hikarigaoka, Nerima-ku, Tokyo, Japan we report an adolescent girl who was diagnosed with IgAN following the first dose of the vaccine.

\section{Case report}

The patient is a previously healthy 17 -year-old girl who presented with a 10-year history of microscopic hematuria. She was initially referred to our hospital following a school urinalysis at age of 7 years old that revealed microscopic hematuria. Because there were no abnormal findings in blood test or ultrasonography, we followed her up twice per year as asymptomatic hematuria. Urinary findings 4 months before the COVID-19 vaccination showed only microscopic hematuria (20-29 red blood cells/high power field (HPF)). Although she had never developed gross hematuria when she had upper respiratory infections or received an influenza vaccine, she presented with gross hematuria and proteinuria (urine protein-creatinine ratio; $0.37 \mathrm{~g} / \mathrm{gCre}$ ) at a routine examination 4 days after receiving the first dose of the 
Pfizer vaccine. She did not recognize that gross hematuria had started because she did not check her urine thoroughly. Serum creatinine was $0.58 \mathrm{mg} / \mathrm{dL}$ and estimated glomerular filtration rate calculated using the fifth-order formula of the Japanese Society of Nephrology was $109 \mathrm{~mL} / \mathrm{min} / 1.73 \mathrm{~m}^{3}$. Her Immunoglobulin (Ig) G, IgA, and IgM levels were normal (IgG $1017.1 \mathrm{mg} / \mathrm{dL}, \operatorname{IgA} 300.5 \mathrm{mg} / \mathrm{dL}$, and $\operatorname{IgM}$ $59.6 \mathrm{mg} / \mathrm{dL}$ ). Complement component 3 (C3) was also nor$\mathrm{mal}(90.7 \mathrm{mg} / \mathrm{dL})$. She had not been exposed to severe acute respiratory syndrome coronavirus 2 (SARS-CoV-2) prior to the vaccination. We performed renal biopsy 2 weeks after the first vaccination. Light microscopy revealed mild mesangial proliferation in some of the 35 glomeruli analyzed, and no endo-capillary or extra-capillary proliferation, sclerosis, or adhesion was observed. Oxford MEST-C classification was M0-E0-S0-T0-C0 (Fig. 1a). Immunofluorescence analysis revealed diffuse mesangial $\operatorname{IgA}$ and $\mathrm{C} 3$ deposits, while deposition of IgG, IgM, C1q or Fibrinogen was not detected (Fig. 1b). Electron microscopy revealed electron-dense deposits in the mesangial lesions (Fig. 1c). Consequently, we diagnosed her with mild IgAN. Gross hematuria was detected after both the first and second doses, although her gross hematuria changed to microscopic hematuria (50-99 red blood cells/HPF) within 1 week of each dose, and proteinuria resolved $(0.05 \mathrm{~g} / \mathrm{gCre})$ spontaneously approximately 10 days after the second dose of the vaccine. Therefore, we opted to observe her without administering medication. She was followed up until 16 days after the second dose of the vaccine, at which point urinalysis revealed microscopic hematuria (30-49 red blood cells/HPF) without proteinuria $(0.07 \mathrm{~g} / \mathrm{gCre})$.

\section{Discussion}

Following the start of the worldwide COVID-19 pandemic, vaccines against the virus have been rapidly developed. Furthermore, some vaccine candidates have already been authorized. However, there are several types of vaccine platforms. For example, the Pfizer and Moderna vaccines are messenger RNA (mRNA) vaccines. mRNA vaccines are a new type of vaccine consisting of mRNA (e.g., that encoding the SARS-Cov-2 spike protein) surrounded by lipid nanoparticles. mRNA vaccines have been reported to generate strong cellular and humoral immune responses and are easily scalable [2]. Clinical trials were established and many studies clarified the efficacy and safety of the Pfizer and Moderna vaccines [3]. Even in adolescents, these mRNA vaccines have an acceptable safety profile and are highly effective against COVID-19 [4, 5]. However, some cases of IgAN flare-up following vaccination against COVID-19 have been reported [1, 6-11]. In 13 cases reported previously,
Fig. 1 Pathological findings (Oxford MEST-C classification was M0-E0-S0-T0-C0). a Minor glomerular abnormalities (periodic acid-Schiff, $\times 200$ ). b Diffuse glomerular mesangial IgA deposits (immunofluorescence analysis). c Electron-dense deposits involving the mesangium (electron microscopy)

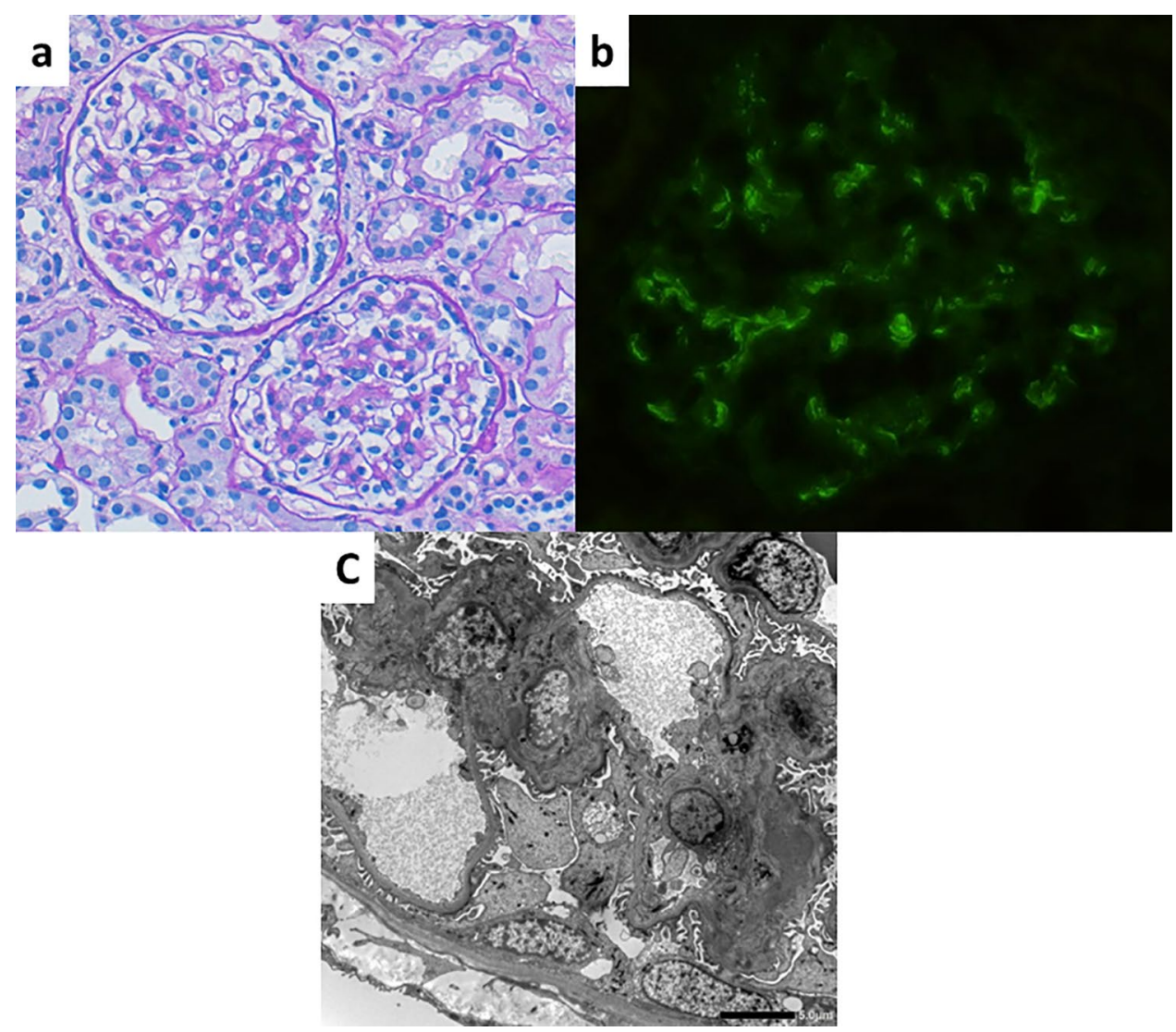


the timing of the appearance of gross hematuria after the first or second vaccine dose ranged from 1 to 5 days (median 1 day; interquartile range; $1-2$ days) [1, 6-11]. Our patient similarly presented with gross hematuria within 4 days of the first vaccine dose. The causation between COVID-19 vaccination and IgAN flare-up remains unclear. There are several reports of IgAN patients presenting with gross hematuria following the second dose of the Pfizer or Moderna vaccines [1, 6-11]. Rahim et al. suggested that a delayed-type hypersensitivity was associated with the IgAN flare [7]. Although most cases of IgAN flare-up occurred after the second vaccine dose, two patients with $\operatorname{IgA}$ vasculitis and one kidney transplant recipient with IgAN developed gross hematuria even after the first dose of the vaccine and without known SARS-CoV-2 exposure, such as in the present case $[8,10]$. Therefore, this side effect is possibly unrelated to the antiSARS-CoV-2 antibody response.

Additionally, as in our patient, there are some cases newly diagnosed with IgAN by observation of new-onset gross hematuria. One had moderate to severe tubulo-interstitial scarring on renal biopsy, which suggested that he had underlying preexisting IgAN that was exacerbated by vaccination [1]. In contrast, in Japan, we are successful at early detection of chronic glomerulonephritis owing to the school urinalysis system. The Japan Society of School Health recommends that doctors follow up patients with asymptomatic hematuria once or twice per year. Therefore, many pediatric nephrologists are following patients with subclinical IgAN that has yet to be diagnosed because of the finding of hematuria alone. As vaccinations progress, some of these patients may ultimately be diagnosed with IgAN because of post-vaccination gross hematuria. Nephrologists should inform both patients with IgAN and those with asymptomatic hematuria that this side effect can occur even after the first dose of the COVID-19 vaccine.

According to the 2020 Japanese Society for Pediatric Nephrology guidelines for pediatric IgA nephropathy [12], renal biopsy should be considered when the patient has proteinuria and hematuria persisting for 6 months or recurrent gross hematuria. However, we performed renal biopsy soon after the first gross hematuria because of the possibility that the patient could not undergo appropriate medical follow-up while studying abroad. Nevertheless, her gross hematuria and proteinuria improved within 2 weeks after the second dose of the vaccine. Most reported cases also recovered spontaneously within a short period of time and without medication $[1,7,8,11]$. Therefore, patients who present with gross hematuria and proteinuria following vaccination against COVID-19 can be observed without performing renal biopsy unless their renal function deteriorates. In contrast to such cases, there are previous reports of IgAN patients who were found to have acute kidney injury following the vaccination and who required medication, such as steroids or renin-angiotensin-aldosterone system inhibitors $[1,8,9]$. Excluding renal transplant and dialysis cases from the reported 13 patients, 3 of the 7 patients with available data on renal function before the $\operatorname{Ig} \mathrm{A}$ flare-up had elevated serum creatinine levels after vaccination $[1,8,11]$. These three patients with acute kidney injury already had persistent severe proteinuria prior to the vaccination. It suggests that patients with severe proteinuria should be more aware of the side effect of the COVID-19 vaccine. However, the IgAN patients who required medication only comprised four of the thirteen reported IgAN cases; two had acute kidney injury, and others were diagnosed with IgAN for the first time [1, $8,9,11]$. Furthermore, in a patient with new-onset IgAN who was administered steroids, an acute exacerbation of the preexisting moderate IgAN had been predicted because of chronic lesions on renal biopsy [1]. Therefore, nephrologists should closely follow up patients who develop glomerulonephritis after vaccination against COVID-19. However, patients who had only asymptomatic microscopic hematuria prior to the vaccination may be less likely to require additional treatment. Finally, we should recognize that there is no need to avoid vaccination more than necessary.

Acknowledgements We thank Richard Robins, PhD, from Edanz (https://jp.edanz.com/ac) for editing a draft of this manuscript.

Author contributions MO was involved in patient care and wrote the manuscript. EK helped perform renal biopsy on the patient and contributed to critical revision of the manuscript. MN, OA, and MS supervised this case and contributed to critical revision of the manuscript. All authors read and approved the final manuscript.

\section{Declarations}

Conflict of interest The authors have declared that no conflict of interest exists.

Human and animal rights This article does not contain any studies with human participants or animals performed by any of the authors.

Informed consent Informed consent was obtained from the patient included in this article.

\section{References}

1. Hanna C, Hernandez LPH, Bu L, Kizilbash S, Najera L, Rheault MN, Czyzyk J, Kouri AM. IgA nephropathy presenting as macroscopic hematuria in 2 pediatric patients after receiving the Pfizer COVID-19 vaccine. Kidney Int. 2021. https://doi.org/10.1016/j. kint.2021.06.032.

2. Pascual-Iglesias A, Canton J, Ortega-Prieto AM, Jimenez-Guadeno JM, Regla-Nava JA. An overview of vaccines against SARSCov-2 in the COVID-19 pandemic era. Pathogens. 2021;10:1030. https://doi.org/10.3390/pathogens10081030.

3. Meo SA, Bukhari IA, Akram J, Meo AS, Klonoff DC. COVID19 vaccines: comparison of biological, pharmacological 
characteristics and adverse effects of Pfizer/BioNTech and moderna vaccines. Eur Rev Med Pharmacol Sci. 2021;23(3):1663-9.

4. Frenck RW Jr, Klein NP, Kitchin N, et al. Safety, immunology, and efficacy of the BNT162b2 COVID-19 vaccine in adolescents. N Engl J Med. 2021;385:239-50.

5. Ali K, Berman G, Zhou H, et al. Evaluation of mRNA-1273 SARS-CoV-2 vaccine in adolescents. N Engl J Med. 2021. https:// doi.org/10.1056/NEJMoa2109522.

6. Negrea N, Rovin BH. Gross hematuria following vaccination for severe acute respiratory syndrome coronavirus 2 in 2 patients with IgA nephropathy. Kidney Int. 2021;99:1487.

7. Rahim SEG, Lin JT, Wang JC. A case of gross hematuria and IgA nephropathy flare-up following SARS-CoV-2 vaccination. Kidney Int. 2021;100:238.

8. Park K, Miyake S, Tai C, Tseng M, Andeen NK, Kung VL. Letter regarding: "a case of gross hematuria and IgA nephropathy flare-up following SARS-Cov-2 vaccination.” Kidney Int Rep. 2021;6:2246-7.

9. Abramson M, Yu SMW, Campbell KN, Chung M, Salem F. IgA nephropathy after SARS-CoV-2 vaccination. Kidney Med. 2021. https://doi.org/10.1016/j.xkme.2021.05.002.
10. Perrin P, Bassand X, Benotmane I, Bouvier N. Gross hematuria following SARS-CoV-2 vaccination in patients with IgA nephropathy. Kidney Int. 2021;100:466-8.

11. Plasse R, Nee R, Gao S, Olson S. Acute kidney injury with gross hematuria and $\operatorname{Ig}$ A nephropathy after COVID-19 vaccination. Kidney Int. 2021. https://doi.org/10.1016/j.kint.2021.07.020.

12. The Japanese Society for Pediatric Nephrology. The 2020 Japanese Society for Pediatric Nephrology guidelines for pediatric IgA nephropathy. Shindan to Chiryo-sya; 2020. https://minds.jcqhc.or. jp/docs/g1_pdf/G0001187/4/iga_nephropathy_in_children.pdf.

Publisher's Note Springer Nature remains neutral with regard to jurisdictional claims in published maps and institutional affiliations. 